\title{
1 How Exciton Interactions Control Spin-Depolarisation in Layered Hybrid Perovskites
}

2 Sean A. Bourelle ${ }^{1 \dagger}$, Ravichandran Shivanna ${ }^{1 \dagger}$, Franco V.A. Camargo ${ }^{2}$, Soumen Ghosh ${ }^{2}$, Alexander J.

3 Gillett $^{1}$, Satyaprasad P. Senanayak ${ }^{1,3}$, Sascha Feldmann ${ }^{1}$, Lissa Eyre ${ }^{1,4}$, Arjun Ashoka ${ }^{1}$, Tim W.J. van de Goor ${ }^{1}$, Haralds Abolins ${ }^{1}$, Thomas Winkler ${ }^{1}$, Giulio Cerullo ${ }^{2}$, Richard H. Friend ${ }^{1}$, Felix Deschler ${ }^{4 *}$

$5 \quad \dagger$ These authors contributed equally

61 Cavendish Laboratory, University of Cambridge, J J Thomson Avenue, Cambridge CB3 OHE, UK

72 IFN-CNR, Dipartimento di Fisica, Politecnico di Milano, Piazza Leonardo da Vinci, 32, 20133,

8 Milano, Italy

93 CSIR-Institute of Minerals \& Materials Technology, Bhubaneswar, India

104 Walter-Schottky-Institute, Physics Department, Technical University Munich, Am Coulombwall 4, 11 Garching bei München, Germany

* Correspondence should be sent to F.D. (felix.deschler@wsi.tum.de)

\section{Abstract}

Using circularly-polarised broadband transient absorption, time-resolved circular photoluminescence, and transient Faraday rotation spectroscopy, we report that spin-dependent interactions have a significant impact on exciton energies and spin depolarisation times in layered Ruddlesden-Popper hybrid metal-halide perovskites. In $\mathrm{BA}_{2} \mathrm{FAPb}_{2} \mathrm{I}_{7}$, we report that room temperature spin lifetimes are largest ( $3.2 \mathrm{ps}$ ) at a carrier density of $\sim 10^{17} \mathrm{~cm}^{-3}$, with increasing depolarisation rates at higher exciton densities. This indicates that many-body interactions reduce spin-lifetimes and outcompete the effect of D'yakonov-Perel precessional relaxation that has been previously reported at lower carrier densities. We further observe a dynamic circular dichroism that arises from a photoinduced polarisation in the exciton distribution between total angular momentum states. Our findings provide fundamental, and application relevant insights into the spin-dependent exciton-exciton interactions in layered hybrid perovskites.

\section{Key Words:}

2D perovskites, many body interactions, spin, exciton, total angular momentum, transient absorption spectroscopy. 
1 Excitons are quasi-particles composed of an electron and hole that are bound together by Coulomb attraction. A detailed picture of the spin-dependent properties and spin relaxation mechanisms of these states in low-dimensional semiconductors is crucial for applications that utilise spin-dependent properties, such as circularly polarised emission in spin-LEDs, circularly polarised laser systems and spin valves. ${ }^{1-5}$ Spintronic semiconductors that incorporate a spin degree of freedom are split between materials with low spin orbit coupling (SOC) for long spin lifetimes, and those with high SOC for efficient spin generation and manipulation. ${ }^{6,7}$

Lead-halide perovskite semiconductors fall into the second category, as orbital angular momentum and spin are coupled by the strong SOC experienced by carriers around the heavy lead and halide atoms that constitute the conduction and valence bands. ${ }^{8-10}$ Since the total angular momentum $J$ is conserved in optical transitions, the selection rule $\Delta m_{j}= \pm 1$ must be fulfilled for circularly polarised photoexcitation. ${ }^{11,12}$ This rule governs the allowed optical transitions between the electron's state of total angular momentum projection $m_{j}= \pm \frac{1}{2}$ in the plane perpendicular to photon propagation. It is important to note that, due to strong SOC, the $J=1 / 2$ electron states are not spin pure $\left(m_{j}= \pm 1 / 2\right.$ total angular momentum projections contain a ratio of $2: 1$ or $1: 2$ spin up:down), while the $S=1 / 2$ hole states are spin pure. ${ }^{12,13}$ The term exciton $J$-polarisation is herein used to refer to the total angular momentum polarisation within the exciton population, although we note that a polarisation in $J$ contains both orbital and spin contributions. Spin relaxation of electrons and holes has been studied in the 3D bulk perovskites, with reports indicating a fast spin flip of the hole $(<1 \mathrm{ps})$ and longer-lived spin polarisation of the electron $(\sim 3 \mathrm{ps}) .{ }^{12}$ In the layered perovskite structure $J$-polarised exciton lifetimes exceeding $1 \mathrm{~ns}$ at $4 \mathrm{~K}$ and quantum beats between excitonic states under an applied magnetic field have been shown. ${ }^{13,14}$ However, many body interactions, which have been shown to play a role in the optical properties of perovskites, have not yet been addressed in the context of spin, despite their expected importance in the presence of strong SOC..$^{15,16}$

The Ruddlesden-Popper layered hybrid perovskites are self-assembled quantum well structures, with dielectric screening that is reduced from the bulk phase by the inclusion of large organic cations between the semiconducting perovskite layers. In such quantum well systems, optical excitation generates high $k$ or lowest energy excitons (with binding energy up to $\sim 300 \mathrm{meV}$ ) for absorbed photon energies below the conduction band, and interacting free charge carriers for excitation into the conduction band. ${ }^{17}$ These excitons and charge carriers are confined to the inorganic layers by organic cations with a low dielectric constant, which explains the particularly large binding energy and Coulomb enhancement factors. ${ }^{18,19}$ At room temperature most carriers rapidly cool to the lowest exciton state and remain bound for the full lifetime of the excited state population. This is in contrast to the 3D metal-halide perovskites, where most resonantly excited excitons quickly dissociate into a dominant population of free 
1 charges. ${ }^{20,21}$ The room temperature depolarisation of the total angular momentum states in layered 2 hybrid perovskite structures has been reported to occur on picosecond timescales, ${ }^{13,22,23}$ but the effect 3 of many body interactions on the depolarisation mechanism remains unclear, and is the focus of this 4 study.

6 Following the optical selection rules set out above, we excite electrons into one of the two optically active $m_{j}$ states by absorption of circularly polarised photons which carry a total spin angular momentum of $l= \pm 1$. We define the resulting exciton $J$-polarisation, $P_{E}$, by

$$
P_{E}=\frac{|+1\rangle-|-1\rangle}{|+1\rangle+|-1\rangle}
$$

9 where $|+1\rangle$ and $|-1\rangle$ are the optically active (bright) exciton states with a total angular momentum of $10 \pm 1$, as determined by the $J=1 / 2$ electron and $S=1 / 2$ hole. ${ }^{12,24}$ A population imbalance between the two exciton states will lead to different absorption of left-handed $\left(\sigma^{-}\right)$and right-handed $\left(\sigma^{+}\right)$circularlypolarised photons - a photoinduced circular dichroism. Here we investigate exciton interactions and spin depolarisation kinetics using three complementary spectroscopies: broadband circularly-polarised transient absorption (CTA), transient grating time-resolved circularly-polarised photoluminescence (CPL), and time-resolved Faraday rotation (FR). We compare the 3D bulk variant $\mathrm{MAPbBr}_{3}$ to the layered variant $(\mathrm{BA})_{2}(\mathrm{FA})_{1} \mathrm{~Pb}_{2} \mathrm{I}_{7}$ (BA, butylammonium; $\mathrm{FA}$, formamidinium), which is composed of 2D inorganic quantum wells, each with a thickness of two unit cells $(n=2)$, and each separated by large organic cations.

\section{Spectral Signatures of $J$-Polarisation within the Exciton Populations}

First, we examine the excited state population dynamics in thin films of the 2D and 3D bulk perovskites via CTA spectroscopy. These measurements are performed using a broadband probe spanning 490-700 $\mathrm{nm}(2.53-1.77 \mathrm{eV})$ and a narrowband pump - tuned from $575 \mathrm{~nm}$ to $490 \mathrm{~nm}(2.16$ to $2.53 \mathrm{eV})$ with 10 $\mathrm{nm}$ FWHM at $575 \mathrm{~nm}\left(37 \mathrm{meV}\right.$ FWHM) - that are set to be polarised either right handed circularly $\left(\sigma^{+}\right)$ or left handed circularly $\left(\sigma^{-}\right)$. Exciton states with total angular momentum, $|+1\rangle$ or $|-1\rangle$, are generated using a $\sigma^{+}$or $\sigma^{-}$polarised pump pulse, respectively. A co-polarised (counter-polarised) probe pulse thus probes the exciton states that are initially occupied (unoccupied) by the pump. 
(a)

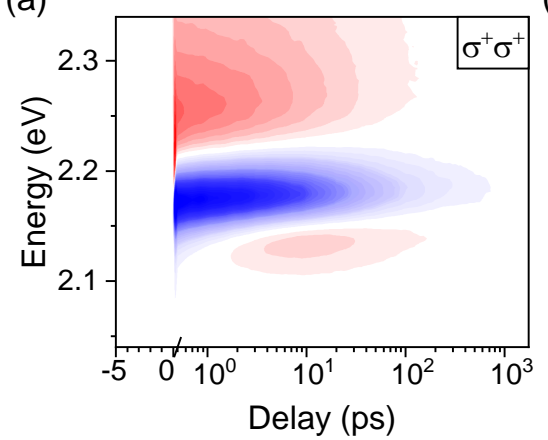

(c)

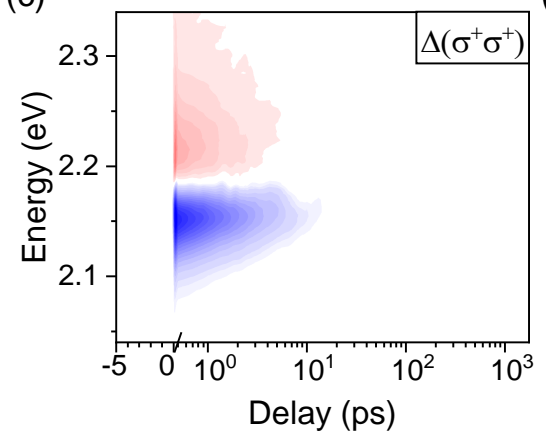

(b)

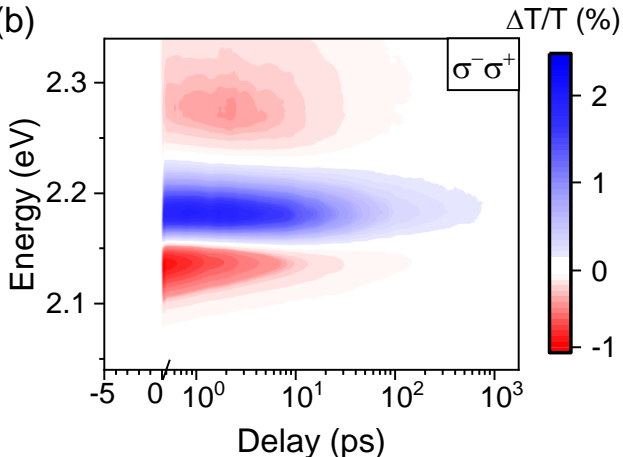

(d)

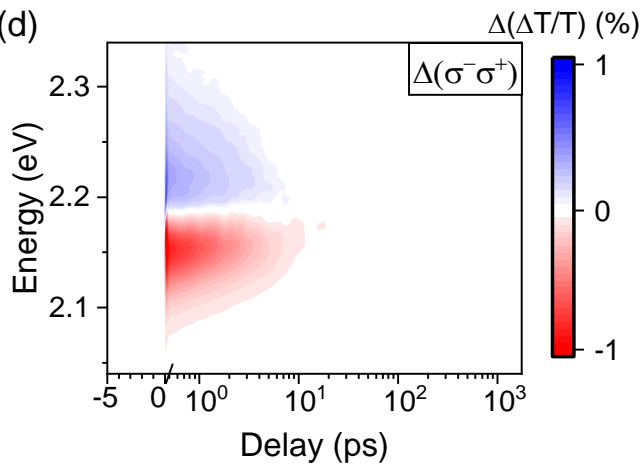

Figure 1: (a) and (b) Contour plots of circularly-polarised transient absorption (CTA) for layered perovskite $\mathrm{BA}_{2} \mathrm{FAP} \mathrm{b}_{2} \mathrm{I}_{7}$ under (a) co-circular and (b) counter-circular pump/probe with a wavelength of $570 \mathrm{~nm}(2.18 \mathrm{eV})$ and fluence of $1 \mu \mathrm{J} \mathrm{cm}$ (initial photoexcited carrier density of $5 \times 10^{17} \mathrm{~cm}^{-3}$ ) (c) and (d) Subtraction of (a) and (b) from a linearly polarised pump TA map. (c) Indicates a blue shift in the energy of the co-polarised states. (d) Indicates a red shift in the energy of the counter-polarised states. Maps plotted on same axis, with same contour intensities for comparison.

The co- and counter-polarised CTA maps for $(\mathrm{BA})_{2} \mathrm{FAPb}_{2} \mathrm{I}_{7}$ in Figure $1(\mathrm{a}, \mathrm{b})$ show the expected exciton ground state bleach (GSB) centred at $2.175 \mathrm{eV}$ - energetically at resonance with the exciton absorption, and $\sim 20 \mathrm{meV}$ higher in energy than the Stokes-shifted PL emission (Supporting Figure S1). Further, we find two $J$-dependent photoinduced absorption (PIA) features, one at energies above the exciton bleach $\left(\mathrm{PIA}_{\mathrm{High}}\right)$ and one below the exciton bleach (PIA $\mathrm{Low}_{\text {Lo }}$, that are indicative of a shift in the absorption peak to higher, and lower energies respectively (Supporting Figure S2).

Figure $1(\mathrm{c}, \mathrm{d})$ present the difference between co/counter-polarised and linear TA spectra (Supporting Figure S2), which highlight the spectral shifts in optical transitions from J-polarized exciton populations. These are extracted by subtracting the co- and counter-polarised CTA maps from a TA map with linearly polarised pump (Supporting Figure S3a). These maps show that the energies of the excitonic states are shifted by the presence of $J$-polarised excitons following photoexcitation: the counter-polarised states, $\sigma^{\mp} \sigma^{ \pm}$(pump polarisation, probe polarisation), are shifted to lower energies, giving rise to PIA $\mathrm{A}_{\text {Low }}$, while the co-polarised states, $\sigma^{ \pm} \sigma^{ \pm}$, are shifted to a higher energy, giving rise

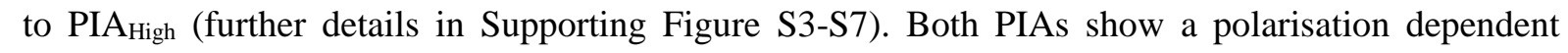


response on picosecond timescales while an exciton $J$-polarisation is present. After $J$-depolarisation, both PIA features remain visible and are equally present under both co- and counter-polarised probing for the duration of the exciton lifetime (Supporting Figure S3b).

These observations have previously been discussed as a broadening of the exciton resonance. ${ }^{25}$ However, Figure 1 shows well-defined spectral shifts, in opposite directions for interacting excitons of equal or opposite $J$ (independent of the pump helicity, Supporting Figure S4). This is inconsistent with a scattering based broadening, which would be symmetric. Thus, the broadening mechanism can now be understood as a $J$ dependent shift in energy of the of the $| \pm 1\rangle$ exciton states following interaction with one another: an energetic splitting of the two-exciton interaction state.

This splitting can be attributed to exciton-exciton exchange interactions, i.e. the mutual repulsion (attraction) between excitons with identical (opposite) J-polarisation, due to the exclusion principle acting separately on the electrons and holes, as recently reported ${ }^{26-29}$ and in agreement with the splitting that has been observed in GaAs quantum wells. ${ }^{30-32}$ This is a similar idea to the bonding and antibonding states of a hydrogen molecule ${ }^{33-35}$. For this reason, a pairing of $|+1\rangle$ and $|-1\rangle$ excitons in real space (corresponding to the counter-polarised probe) lowers the energy of both exciton species, while a pairing of two $|+1\rangle$ (or two $|-1\rangle$ ) excitons (corresponding to the co-polarised probe) will raise their respective energies. ${ }^{26,27}$ This observation is consistent with previous reports of stable bi-exciton formation with $44 \mathrm{meV}$ binding energy in layered hybrid perovskites with $\mathrm{n}=1$ layer number, and agrees with the observation of repulsive exciton pair states and exciton fine structure. ${ }^{36,37}$ Further, our observation of a $15 \pm 3 \mathrm{meV}$ exciton interaction energy (Supporting Figure S2 and S5) is consistent with other reports for this material. ${ }^{15}$

By reducing the exciton density, many body exciton interactions will be reduced. In the 3D perovskite $\mathrm{MAPbBr}_{3}$, the BA cations are replaced with smaller MA cations. This minimizes the quantum confinement of excitations and, hence, reduces their excitonic character-lowering the exciton binding energy to $<40 \mathrm{meV}$ as compared to the $300 \mathrm{meV}$ in the $2 \mathrm{D}$ layered perovskite. ${ }^{38,39}$ Thus, most excitations in $\mathrm{MAPbBr}_{3}$ evolve to free carriers at room temperature, with primary exciton dissociation occurring within the first $\sim 200 \mathrm{fs} .^{20,40,41}$ We therefore expect to find a significant reduction in both PIA $_{\text {Low }}$ and PIA $\mathrm{H}_{\text {High }}$ due to their origin from exciton interactions.

Figures 2(a) and 2(b) show CTA measurements on $\mathrm{MAPbBr}_{3}$ which are dominated by a GSB (blue) and PIA (similar to that reported in $\mathrm{MAPbI}_{3}{ }^{42}$ ). As in the layered perovskites, we find $J$ dependent signals of PIA $\mathrm{L}_{\mathrm{Low}}$ and PIA $\mathrm{High}_{\text {that }}$ persist after depolarisation for the full lifetime of the excited state population, but with a significant reduction in strength by $\sim 80 \%$ compared to the layered hybrid perovskite. This is highlighted in Figure 2(c), which compares the initial CTA spectra of the populated state (co- 
1 polarised probe, thus with very few counter polarised charge carriers) to the spectra after exciton $J$ -

2 depolarisation (integrated 20-100 ps after excitation), at which time we expect to observe PIA $\mathrm{A}_{\text {Low }}$ due

3 to the presence of counter polarised excitons. We find a small decrease of the signal on the red side of

4 the GSB of $\mathrm{MAPbBr}_{3}$, which we attribute to $\mathrm{PIA}_{\text {Low }}$ from a non-geminate exciton population, as

5 discussed above for the layered perovskite. Importantly, the significantly reduced PIA Low $_{\text {intensity in }}$

6 the 3D perovskite sample (with respect to the GSB peak) agrees with the reduced exciton population

7 that is expected due to the smaller exciton binding energy. ${ }^{43-45}$ This is further evidenced by

8 measurements on $\mathrm{MAPbI}_{3}$ (Supporting Figure S8) which has even lower exciton binding energy. In

9 summary, we observe a $J$ dependent shift in energy of the of the $| \pm 1\rangle$ exciton states due to exciton-

10 exciton interactions in both bulk and layered perovskites following photoexcitation. A sketch

11 summarizing the electronic shifts in energy levels due to these interactions is presented in Figure 2 (d).

12 Following the photoexcitation of excitons, new transitions into the bonding/antibonding states become

13 possible, with the relative transition strength dependent on the population of the $|+1\rangle$ and $|-1\rangle$

14 exciton states.

(a)

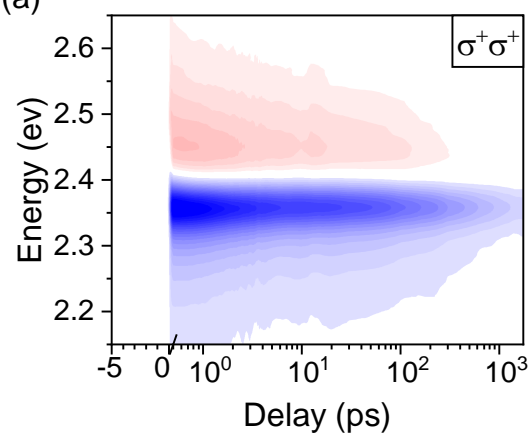

(c)

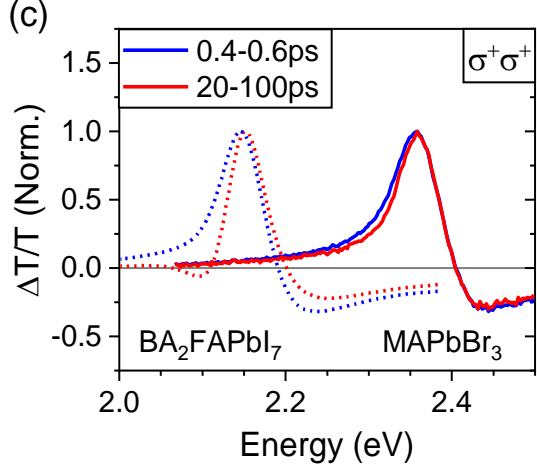

(b)

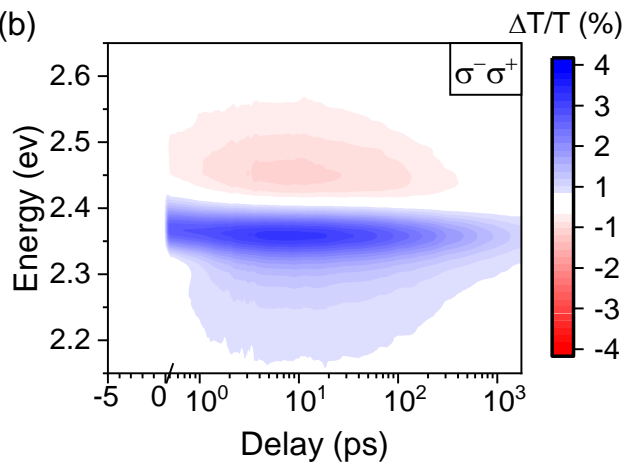

(d)

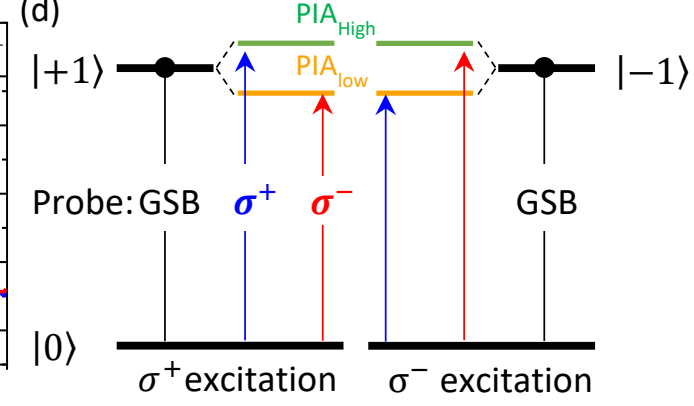

Figure 2: CTA contour plots for bulk perovskite $\mathrm{MAPbBr}_{3}$ under (a) co-polarised probe and (b) counter-polarised probe, at a pump wavelength of $500 \mathrm{~nm}(2.48 \mathrm{eV})$ and fluence of $3 \mu \mathrm{J} \mathrm{cm}{ }^{-2}$. (c) Comparison of early and long-time circularly co-polarised transient absorption spectra for layered $\mathrm{BA}_{2} \mathrm{FAPbI}_{7}$ (dotted) and bulk MAPbBr 3 (solid) perovskites. (d) Schematic depicting the shifts in the exciton levels after optical excitation as a result of the exchange interaction between excitons. PIA High $\left(P I A_{\text {Low }}\right)$ is the dominant transition at $t=0 \mathrm{ps}$ for probe pulses co-(counter-) polarised to the pump, as indicated by blue and red arrows. 

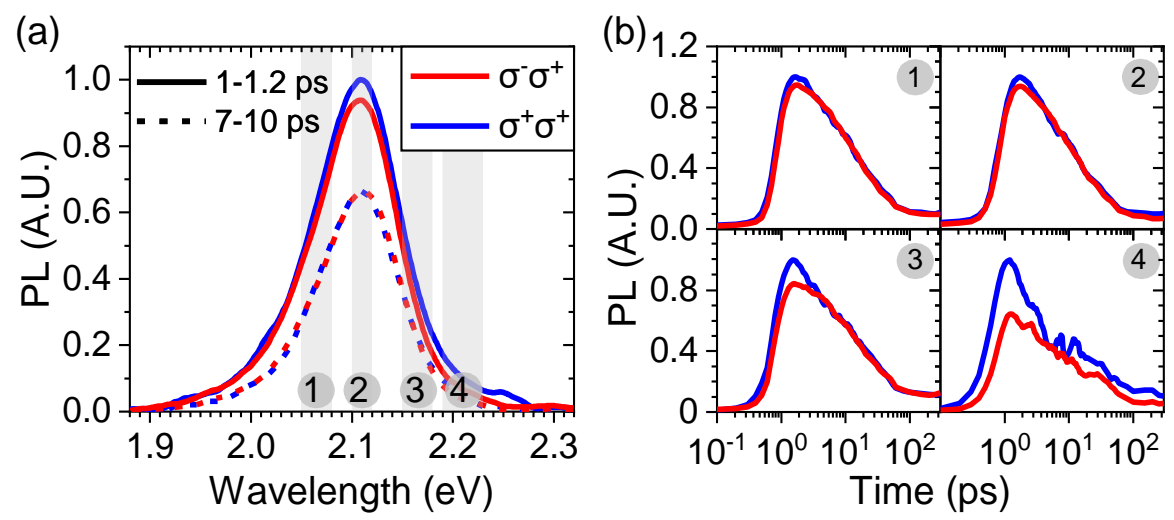

Figure 3: Time resolved CPL spectra of layered perovskite $\mathrm{BA}_{2} \mathrm{FAPb}_{2} \mathrm{I}_{7}$ samples for $\sigma^{+}$detection under

$3 \sigma^{-}$or $\sigma^{+}$excitation at $500 \mathrm{~nm}(2.48 \mathrm{eV}), 2 \mu \mathrm{Jcm}^{-2}$ with a $200 \mathrm{fs}$ laser pulse. Normalised by maximum

4 of co-polarised emission. (a) Emission spectrum for detection co- and counter-polarised to excitation

5 at time delays before and after depolarisation. ${ }^{46}$ (b) Kinetics of the CPL emission integrated over four

6 spectral ranges, showing a difference in the degree of polarisation.

7 In contrast to chiral perovskites, CPL from hybrid perovskites must arise from a polarisation in the

8 carrier distribution. Thus, observing steady state CPL requires spin lifetimes that are sufficiently long

9 in comparison with the radiative recombination lifetime. Here, we successfully detect and analyse

10 these processes by employing a transient-grating PL technique (details in Methods section), which has

11 a temporal resolution around $150 \mathrm{fs}$. Figure 3 (a) compares the early time spectra of CPL for the 2D

12 system at $1 \mathrm{ps}$ and at $\sim 10 \mathrm{ps}$, after depolarisation has occurred. The emission centre is located near

$132.12 \mathrm{eV}, \sim 60 \mathrm{meV}$ red-shifted from the exciton level observed in TA, and $40 \mathrm{meV}$ lower in energy

14 than that observed in steady state emission. This discrepancy is within our calibration accuracy.

15 Figure 3 (b) demonstrates the depolarisation kinetics integrated over the four different energy regions

16 of the CPL spectrum indicated in Figure 3 (a). We observe that the blue side of the CPL spectra is

17 initially more strongly polarised. This is expected from our previous analysis. Blue shifted emission is only expected for interacting co-polarised excitons. This is most probable when the polarisation is at a maximum, i.e. at $\mathrm{t}=0$ after photoexcitation. Counter polarised exciton interactions are most probable once the exciton population has depolarised.

Further, the depolarisation kinetics of the co- and counter-polarised emission spectra match the polarisation timescales observed in CTA, which are further discussed in the next section (Supporting Figure S9). To our knowledge, this is the first observation of room temperature CPL in non-chiral layered hybrid perovskites and confirms that a polarisation in the excited exciton population leads to polarised emission. 


\section{Analysis of Mechanisms Limiting Exciton Depolarisation Times}

2 Our observations indicate a driving force towards spin flip upon exciton-exciton scattering in the layered perovskites. As the photoexcited excitons are localised charge carriers, the probe pulses specifically interrogate regions of the material where excitons are present. We have shown that the counter polarised transition requires less energy. Thus, when two exciton wave functions overlap, J-flip into the anti-parallel $J$ configuration is energetically favoured. We use FR and CTA measurements to determine the effects of such exciton-exciton scattering on the room temperature depolarisation kinetics.

We analyse the exciton depolarisation kinetics between the two eigenstates $|+1\rangle$ and $|-1\rangle$ from the difference between the co- and counter-polarized CTA spectra, spectrally integrated around PIA $\mathrm{Low}_{\text {, and }}$ normalised by the total GSB signal. We adapt the definition of $P_{E}$ from Equation 1 to track changes in the exciton polarisation using the definition of $P_{E}^{*}$ below:

$$
P_{E}^{*}(t)=\frac{\left.\left[\frac{\Delta T}{T}\left(\sigma_{\text {pump }}^{+}\right)-\frac{\Delta T}{T}\left(\sigma_{\text {pump }}^{-}\right)\right]\right|_{\Delta \mathrm{E}(\mathrm{t})}}{\left.\left[\frac{\Delta T}{T}\left(\sigma_{\text {pump }}^{+}\right)+\frac{\Delta T}{T}\left(\sigma_{\text {pump }}^{-}\right)\right]\right|_{G S B(t)} .}
$$

14

Here, $T$ is the probe transmission. The dichroism at a time, $t$, is integrated over a consistent energy range, $\Delta E=2.08-2.10 \mathrm{eV}$, and normalised to the total transmission change at the GSB. This formula is used to track changes in the exciton polarisation, rather than to quantify the absolute polarisation, $P_{E}$. The absolute value of $P_{E}$ cannot be extracted from the GSB due to the spectral shifts described above that dominate over phase space filling, (details in Supporting Information, Section 2).

As the pump photon energy is increased with respect to the exciton peak, we observe a rise time in $P_{E}^{*}$ (Figure 4a). This is due to an increase in the exciton cooling time, ${ }^{42,47,48}$ as the CTA signal in the region $\Delta E$ increases during exciton cooling $-P_{E}^{*}$ is determined from the thermalized exciton population. Further, we observe a more rapid decrease in the value of $P_{E}^{*}$ during cooling (Figure 4b). After cooling, the depolarisation kinetics are independent of excitation energy and the exciton $J$-depolarisation time, $\tau$, obtained from mono-exponential fit to the $P_{E}^{*}$ decay, yields 2.5 ps at a photoexcited carrier density of $\sim 10^{18} \mathrm{~cm}^{-3}$, irrespective of the excitation energy (Figure 4a, inset, and Supporting Figure S10). These observations, made following the photoexcitation of excitons with increased kinetic energy, can be explained by both the D'yakonov Perel (DP) and Elliott Yafet (EY) mechanisms. In the DP mechanism, the increase in the exciton's crystal momentum increases the effective magnetic field around which spins precess. This increases the rate of precession and, correspondingly, the depolarisation rate. ${ }^{49}$ In an EY dominated regime, the increased scattering rates (due to increased crystal momentum and exciton 
1 Bohr radius) will similarly increase spin relaxation. ${ }^{50}$ For carrier density below $10^{16} \mathrm{~cm}^{-3}$, Todd, Riley 2 et.al. have excluded EY dominated spin relaxation. ${ }^{49}$
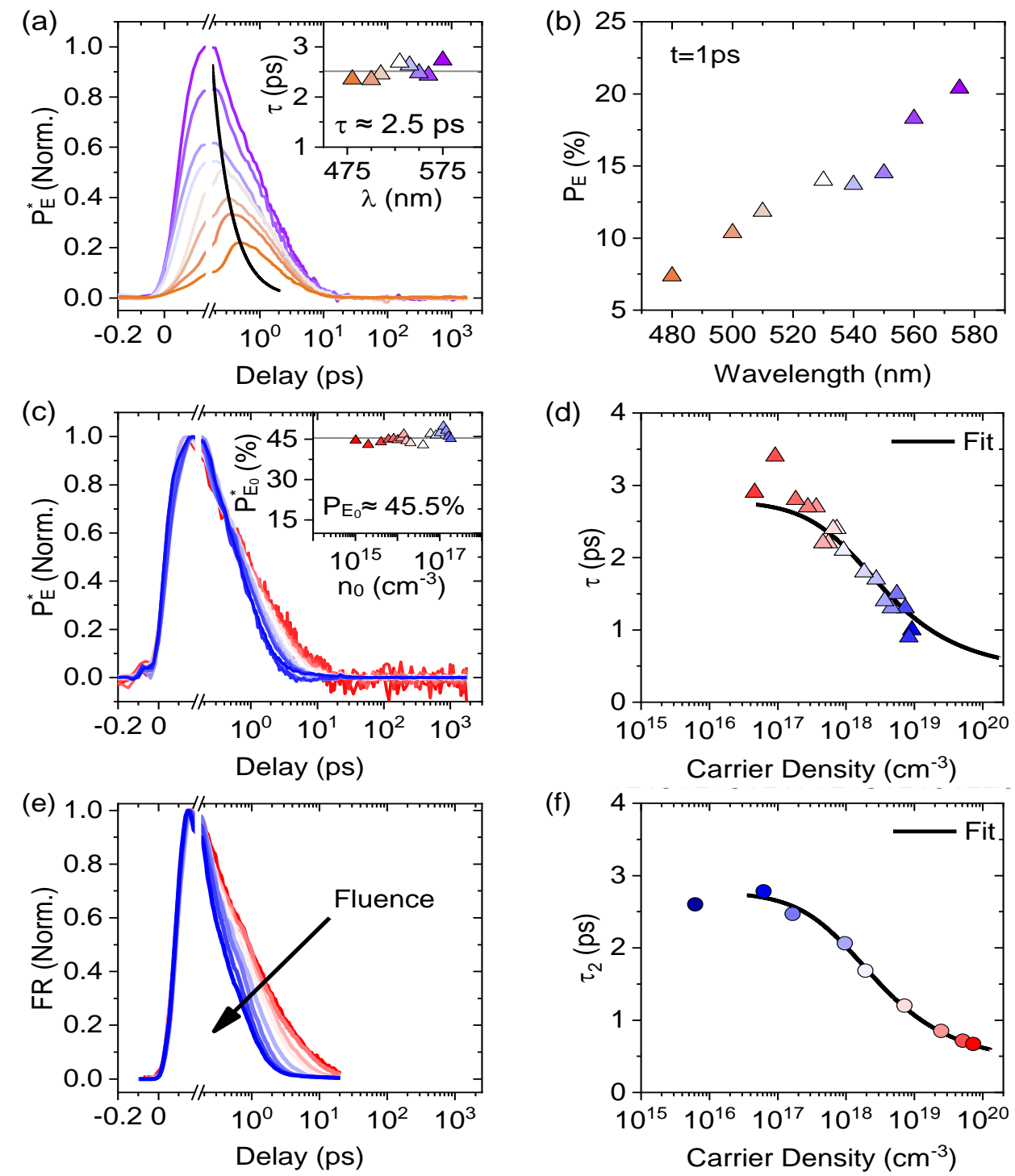

4 Figure 4: Exciton J-polarisation $P_{E}^{*}$ for $B A_{2} F A P b_{2} I_{7}$. (a) Depolarisation kinetics for different excitation

6 fluence of $2 \mu \mathrm{J} \mathrm{cm}^{-2}$. Kinetics are normalised to the value of $P_{E}^{*}(t=200 \mathrm{fs})$ under resonant $7 \quad(575 \mathrm{~nm} / 2.15 \mathrm{eV})$ excitation. The exciton depolarisation time, $\tau$, is obtained from a mono-exponential 8 decay fit for $t>1 \mathrm{ps}$ and is independent of pump energy (inset). (b) Dependence of $P_{E}^{*}(t=1 \mathrm{ps}$ ) on excitation wavelength. (c) Normalised $P_{E}^{*}$ kinetics for increasing fluence at resonant excitation (575 $\mathrm{nm})$. Value of $P_{E}^{*}(t=1 \mathrm{ps})$ is independent of pump fluence (inset). (d) $P_{E}^{*}$ decay times obtained from mono-exponential decay fits to $(c)$. The exciton depolarisation times decrease with increasing fluence. (e) Fluence series and $(\boldsymbol{f}) \tau_{2}$ decay coefficients from bi-exponential fits of Faraday rotation kinetics. The same curve is plotted in $(d, f)$ and is obtained by fitting to an inverse square root dependence on carrier density to the FR data shown in (e). 
1 To isolate the impact of exciton-exciton scattering on the room temperature spin lifetime, we perform

2 fluence dependence CTA measurements under resonant excitation, thus ensuring that the photoinduced

3 phonon population is small (Supplementary Calculation 1). We extend the fluence series carried out by

4 Todd, Riley et al. beyond a carrier density of $10^{16} \mathrm{~cm}^{-3} \cdot{ }^{49}$ Contrary to the observations at lower carrier

5 densities, our fluence series reveals that increasing carrier density above $10^{17} \mathrm{~cm}^{-3}$ increases the rate of

6 exciton depolarisation, while leaving the initial polarisation unchanged (Figure 4c, inset). The excitation

7 photon energy was set slightly below the excitonic peak in these experiments to minimize effects from

8 phonon scattering. The exciton depolarisation time is obtained from a mono-exponential fit and plotted

9 as a function of the initial photoexcited carrier density $n$ (Figure 4d). Similar observations have been

10 made in GaAs quantum wells, ${ }^{51}$ where the Bir-Aronov Pikus mechanism was found to dominate spin

11 relaxation. Empirically, we describe the carrier dependence of the depolarisation time with a function

$12 \tau=C+a\left(n+n_{c}\right)^{-0.5}$ where $n_{c}$ is a critical carrier density and $C \sim 0.5$ ps is the limit of the

13 depolarisation time at high carrier densities. Considering the results presented by Todd, Riley et al., we

14 find a maximum lifetime of $3.2 \mathrm{ps}$ for an initial carrier density of $1 \times 10^{17} \mathrm{~cm}^{-3}$. ${ }^{49}$ The observed increase

15 in depolarisation rate at higher carrier density agrees with our earlier analysis that exciton-exciton

16 interactions make it energetically favourable for a flip of the exciton $J$-state to occur. Additionally, it

17 suggests competing mechanisms that have a different dependence on carrier density: i) D'yakonov-

18 Perel type precessional depolarisation that dominates at low carrier densities, ${ }^{49}$ and ii) an excitoninteraction induced depolarisation which dominates at higher densities, and may occur through BirAronov Pikus, as seen in GaAs quantum wells, ${ }^{16,51}$ or EY scattering mechanisms. This could explain the discrepancy between theory and experiment that has previously been discussed, ${ }^{13}$ and reveals the significance of many body interactions on the exciton spin relaxation time in the 2D hybrid perovskite materials.

Unlike TA, which is sensitive to the total angular momentum states, FR measurements are sensitive to the net spin polarisation (Supporting Figure S11). ${ }^{52}$ Our CTA results are confirmed by fluence dependent FR measurements, highlighting that the spin polarisation decays alongside the total angular momentum polarisation (Figure 4e,f). For future spintronic devices that incorporate layered hybrid perovskites, these results indicate an optimal carrier density between $10^{16}$ and $10^{17} \mathrm{~cm}^{-3}$ in order to achieve the longest $J$-depolarisation times. This is a manageable carrier density for current devices such as LEDs. ${ }^{53-55}$

\section{Conclusions}

We report that the excitonic states of layered hybrid perovskites show J-dependent energetic shift following interaction with one another. This explains the origin of the PIA features that have been observed in the layered perovskites, which have often been discussed simply as a state broadening and 
1 is in agreement with previous reports of many body interactions in similar materials ${ }^{15}$. Further, we have 2 shown that exciton-exciton scattering plays an important role in exciton depolarisation. We report that 3 the D'yakonov-Perel type relaxation mechanism previously identified at low carrier densities is 4 outcompeted by depolarisation driven by exciton interactions when the carrier density increases above $510^{17} \mathrm{~cm}^{-3}$. Our results indicate that exciton interactions introduce a driving force towards anti-aligned 6 projections of the total angular momentum and increase the rate of depolarisation, which is a potential 7 mechanism for obtaining high luminescence yields as it may limit the dark $J=0$ exciton population. 8 Lastly, we highlight that there is an optimal carrier density between $10^{16}$ and $10^{17} \mathrm{~cm}^{-3}$ that should be 9 employed in devices aiming to utilise spin states in layered hybrid perovskites. 


\section{Methods}

The excited state carrier dynamics are examined via, CTA. These measurements are performed using a broadband probe (490-700 nm) and a monochromatic pump (tuned from $575 \mathrm{~nm}$ to $490 \mathrm{~nm}$ with $10 \mathrm{~nm}$ FWHM at $575 \mathrm{~nm}$ ). Exciton states with opposite total angular momentum, $|+1\rangle$ or $|-1\rangle$, are generated using a $\sigma^{+}$or $\sigma^{-}$polarised pump. A co-polarised (counter-polarised) beam is then used to probe the initially occupied (unoccupied) exciton level. CTA spectra for co- and counter-polarised pump-probe pulses are obtained by rotating the pump polarisation. All three polarization configurations (co-polarized, counter-polarized, linear) are taken in the same measurement series, by rotating a quarter waveplate in the pump beam between $0^{\circ}, 45^{\circ}, 90^{\circ}$, and, for confirmation of linearity, $-45^{\circ}$. The pump intensity was kept constant for the different polarizations. The polarization maps of Figure $1 \mathrm{c} / \mathrm{d}$ were calculated by simple subtraction of the circularly pumped measurements from the linearly pumped one. The transmitted probe beam is dispersed in a monochromator and detected using a CCD array. The change in transmission, $\frac{\Delta T}{T}$, is calculated as a function of wavelength and pump-probe delay using

$$
\frac{\Delta T}{T}=\frac{T_{o n}-T_{o f f}}{T_{o f f}}
$$

where $T_{\text {off }}$ and $T_{o n}$ are the transmission signals of the probe when the pump has been blocked or unblocked by an optical chopper. The pump-probe delay is increased up to $1.5 \mathrm{~ns}$ using an automated delay stage, and the bandwidth of the laser $(10 \mathrm{~nm}$ at $575 \mathrm{~nm})$ is sufficient to encompass the entire exciton resonance. Pulse duration sets the temporal resolution at $<150 \mathrm{fs}$.

The transient grating time resolved photoluminescence spectroscopy is used to analyse the photoluminescence (PL) kinetics following excitation from a circularly polarised pulse. This method was first reported by Chen et. al. ${ }^{56}$ The PL is collected by a spherical mirror and collimated. It is then sent through a quarter waveplate and linear polariser that are set to transmit PL that is co-polarised to the pump pulse of the initial measurement, $\sigma^{+}$. Counter polarised PL transients are collected in the same way by rotating the pump polarisation from $\sigma^{+}$to $\sigma^{-}$before the sample. After polarisation filtering, the PL is sent through a transient grating where it is diffracted. In order to achieve the transient grating, two $800 \mathrm{~nm}$ gating beams are overlapped in time and space onto the surface of fused silica. The interfering beams give rise to an interference pattern that modulates the refractive index of the fused silica, creating a grating that only exists for the pulse duration. ${ }^{56}$ This allows a temporal slice of the PL decay to be spatially separated from the rest; it's temporal width is determined by the time window over which the transient grating exists. The diffracted light is then sent through a spectrometer and onto an ICCD camera. 
The transient Faraday rotation experiments were performed using a regenerative amplified Ti:Sapphire system operating at $2 \mathrm{kHz}$. The pump and probe beams were generated using home-made optical parametric amplifiers at $515 \mathrm{~nm}$ (pump) and $630 \mathrm{~nm}$ (probe, below the bandgap), both with bandwidth of $10 \mathrm{~nm}$. A quarter waveplate immediately before the sample was used to circularly-polarize the pump beam, while the probe is linearly polarized. The pump was chopped at $1 \mathrm{kHz}$, and the probe was detected using a pair of balanced photodiodes placed on a rotation mount after a Wollaston prism pair, such that by rotating the mount the linearly polarized probe can be perfectly split in half between the photodiodes at a negative pump probe time delay. Finally, a lock-in amplifier was used to detect the difference between the signal at the photodiodes with pump on and with pump off, effectively measuring the rotation of the polarization of the probe induced by the circularly polarized pump. The alignment of the setup was tested by comparing the signal of the same sample with right-handed versus left-handed polarization of the pump: a well-aligned setup results in the same signal in both cases, except for a multiplicative factor of minus one (see Supporting Figure S11).

Polycrystalline films of 2D halide perovskites are fabricated following the general formula of $A_{2} B_{n-1} P b_{n} I_{3 n+1}$, where $A$ = butylammonium, $B$ = formamidinium/methylammonium/caesium, and $n=2$ or $\infty$ (bulk $\mathrm{MAPbBr}_{3}$ ) perovskites (Studies on the variants not reported in the main text yield identical results and can be found in the Supplementary Information Section III). Lead iodide, butylammonium iodide, and Formamidinium/ methylammonium /caesium iodide are dissolved in Dimethylformamide at $0.1 \mathrm{M}$ concentration and ratio of 2:2:1. Glass coverslips were sonicated in first acetone, then isopropanol for 5 minutes, before 10 minutes of oxygen plasma etching. The precursor solution was then spin coated at 2000 r.p.m. for $60 \mathrm{~s}$, followed by annealing at $100{ }^{\circ} \mathrm{C}$ for $60 \mathrm{~s} \mathrm{inside}$ the nitrogen filled glovebox. All chemicals are procured from Sigma-Aldrich.

\section{Acknowledgements}

S.A.B acknowledges the support from the EPSRC Centre for Doctoral Training in Graphene Technology (EP/L016087/1). R.S. and R.H.F. acknowledge support from EPSRC project 'Strategic University Network to Revolutionise Indian Solar Energy-SUNRISE (EP/P032591/1)'. R.S. acknowledges the Royal Society Newton-Bhabha International Fellowship. S.P.S acknowledges Royal Society for the Newton Fellowship and funding from the Engineering and Physical Sciences Research Council (EPSRC, grant number: EP/M005143/1). S.G. and G.C. acknowledge the Marie Curie actions (project H2020- MSCA-IF- 2018841356). S.F. acknowledges funding from the Studienstiftung des deutschen Volkes and EPSRC, as well as support from the Winton Programme for the Physics of Sustainability. F.D acknowledges a Winton Advanced Research Fellowship and funding from the DFG Emmy Noether Program.

\section{Author Contributions}


1 S.A.B, R.S. and F.D planned the experiments that were carried out by R.S, S.A.B, F.C, and S.G. F.D, 2 T.W, R.H.F and G.C supervised. A.G, S.P.S , S.F, L.E, A.A, T.W.J van de Goor and H.A provided 3 discussion and thoughts. R.S drafted the results and S.A.B wrote the manuscript with feedback from all 4 authors. 


\section{References}

1. Zutic, I., Fabian, J. \& Sarma, S. Das. Spintronics: Fundamentals and applications. Reviews of Modern Physics 76, 323-410 (2004).

2. Giovanni, D. et al. Tunable room-temperature spin-selective optical Stark effect in solutionprocessed layered halide perovskites. Sci. Adv. 2, (2016).

3. Amo, A. et al. Exciton-polariton spin switches. Nat. Photonics 4, 361 (2010).

4. Nishizawa, N., Nishibayashi, K. \& Munekata, H. Pure circular polarization electroluminescence at room temperature with spin-polarized light-emitting diodes. Proc. Natl. Acad. Sci. 114, 1783-1788 (2017).

5. Holub, M., Shin, J., Saha, D. \& Bhattacharya, P. Electrical spin injection and threshold reduction in a semiconductor laser. Phys. Rev. Lett. 98, (2007).

6. Cummings, A. W., Valenzuela, S. O., Ortmann, F. \& Roche, S. Graphene spintronics. in $2 D$ Materials: Properties and Devices 9, 197-218 (Nature Publishing Group, 2017).

7. Jansen, R. Silicon spintronics. Nature Materials 11, 400-408 (2012).

8. Even, J., Pedesseau, L., Jancu, J. M. \& Katan, C. Importance of spin-orbit coupling in hybrid organic/inorganic perovskites for photovoltaic applications. J. Phys. Chem. Lett. 4, 2999-3005 (2013).

9. Even, J., Pedesseau, L. \& Katan, C. Analysis of Multivalley and Multibandgap Absorption and Enhancement of Free Carriers Related to Exciton Screening in Hybrid Perovskites. J. Phys. Chem. C 118, 11566-11572 (2014).

10. Niesner, D. et al. Giant Rashba Splitting in $\mathrm{CH} 3 \mathrm{NH} 3 \mathrm{PbBr} 3$ Organic-Inorganic Perovskite. Phys. Rev. Lett. 117, (2016).

11. Li, J. \& Haney, P. M. Optical spintronics in organic-inorganic perovskite photovoltaics. Phys. Rev. B 93, 155432 (2016).

12. Giovanni, D. et al. Highly spin-polarized carrier dynamics and ultralarge photoinduced magnetization in CH3NH3PbI3 perovskite thin films. Nano Lett. 15, 1553-1558 (2015).

13. Odenthal, P. et al. Spin-polarized exciton quantum beating in hybrid organic-inorganic perovskites. Nat. Phys. 13, 894-899 (2017).

14. Belykh, V. V et al. Coherent spin dynamics of electrons and holes in $\mathrm{CsPbBr} 3$ perovskite crystals. doi:10.1038/s41467-019-08625-Z

15. Elkins, M. H. et al. Biexciton Resonances Reveal Exciton Localization in Stacked Perovskite Quantum Wells. J. Phys. Chem. Lett 8, 46 (2017).

16. Lü, C., Cheng, J. L. \& Wu, M. W. Hole spin dephasing in p -type semiconductor quantum wells. Phys. Rev. B - Condens. Matter Mater. Phys. 73, (2006).

17. Elliott, R. J. Introduction to the Theory of Excitons. in Polarons and Excitons in Polar 
Semiconductors and Ionic Crystals 271-292 (Springer US, 1984). doi:10.1007/978-1-46132693-9_8

18. Stoumpos, C. C. et al. Ruddlesden-Popper Hybrid Lead Iodide Perovskite 2D Homologous Semiconductors. Chem. Mater. 28, 2852-2867 (2016).

19. Ishihara, T., Takahashi, J. \& Goto, T. Exciton state in two-dimensional perovskite semiconductor (C10H21NH3)2PbI4. Solid State Commun. 69, 933-936 (1989).

20. Jha, A. et al. Direct Observation of Ultrafast Exciton Dissociation in Lead Iodide Perovskite by 2D Electronic Spectroscopy. ACS Photonics 5, 852-860 (2018).

21. Ghosh, T., Aharon, S., Etgar, L. \& Ruhman, S. Free Carrier Emergence and Onset of ElectronPhonon Coupling in Methylammonium Lead Halide Perovskite Films. J. Am. Chem. Soc. 139, $18262-18270$ (2017).

22. Chen, X. et al. Impact of Layer Thickness on the Charge Carrier and Spin Coherence Lifetime in 2D Layered Perovskite Single Crystals. ACS Energy Lett. 3, acsenergylett.8b01315 (2018).

23. Yin, J. et al. Layer-Dependent Rashba Band Splitting in 2D Hybrid Perovskites. Chem. Mater. 30, 8538-8545 (2018).

24. Fiederling, R. et al. Injection and detection of a spin-polarized current in a light-emitting diode. Nature 402, 787-790 (1999).

25. Zhai, Y. et al. Giant Rashba splitting in 2D organic-inorganic halide perovskites measured by transient spectroscopies. Sci. Adv. 3, e1700704 (2017).

26. Yu, Z. G. Effective-mass model and magneto-optical properties in hybrid perovskites. Sci. Rep. 6, 28576 (2016).

27. Becker, M. A. et al. Bright triplet excitons in caesium lead halide perovskites. Nature 553, 189-193 (2018).

28. Combescot, M. The inconstant world of composite excitons. Superlattices Microstruct. 43, 394-398 (2008).

29. Combescot, M. \& Betbeder-Matibet, O. Time evolution of two ground-state excitons. doi:10.1103/PhysRevB.81.235203

30. Damen, T. C., Via, L., Cunningham, J. E., Shah, J. \& Sham, L. J. Subpicosecond spin relaxation dynamics of excitons and free carriers in GaAs quantum wells. Phys. Rev. Lett. 67, 3432-3435 (1991).

31. Stark, J. B., Knox, W. H. \& Chemla, D. S. Spin-resolved femtosecond magnetoexciton interactions in GaAs quantum wells. Phys. Rev. B 46, 7919-7922 (1992).

32. Crooker, S. A., Awschalom, D. D. \& Baumberg, J. J. Optical spin resonance and transverse spin relaxation in magnetic semiconductor quantum wells. Phys. Rev. B - Condens. Matter Mater. Phys. 56, 7574-7588 (1997). 
33. Geiregat, P. et al. Thermodynamic Equilibrium between Excitons and Excitonic Molecules Dictates Optical Gain in Colloidal CdSe Quantum Wells. J. Phys. Chem. Lett 10, 14 (2019).

34. Combescot, M., Betbeder-Matibet, O. \& Dubin, F. Mixture of composite-boson molecules and the Pauli exclusion principle. doi:10.1103/PhysRevA.76.033601

35. Combescot, M., Betbeder-Matibet, O. \& Dubin, F. The many-body physics of composite bosons. Physics Reports 463, 215-320 (2008).

36. Thouin, F. et al. Stable biexcitons in two-dimensional metal-halide perovskites with strong dynamic lattice disorder. Phys. Rev. Mater. 2, (2018).

37. Thouin, F. et al. Phonon coherences reveal the polaronic character of excitons in twodimensional lead halide perovskites. Nat. Mater. 18, 349-356 (2019).

38. Yang, Y. et al. Low surface recombination velocity in solution-grown $\mathrm{CH} 3 \mathrm{NH} 3 \mathrm{PbBr} 3$ perovskite single crystal. Nat. Commun. 6, (2015).

39. Tilchin, J. et al. Hydrogen-like Wannier-Mott Excitons in Single Crystal of Methylammonium Lead Bromide Perovskite. ACS Nano 10, 6363-6371 (2016).

40. Deschler, F. et al. High photoluminescence efficiency and optically pumped lasing in solutionprocessed mixed halide perovskite semiconductors. J. Phys. Chem. Lett. 5, 1421-1426 (2014).

41. Valverde-Chávez, D. A. et al. Intrinsic femtosecond charge generation dynamics in single crystal CH3NH3PbI3. Energy Environ. Sci. 8, 3700-3707 (2015).

42. Price, M. B. et al. Hot-carrier cooling and photoinduced refractive index changes in organicinorganic lead halide perovskites. Nat. Commun. 6, 8420 (2015).

43. Cingolani, R. et al. Radiative recombination processes in wide-band-gap II-VI quantum wells: the interplay between excitons and free carriers. J. Opt. Soc. Am. B 13, 1268 (1996).

44. Wang, W. et al. Interplay between Exciton and Free Carriers in Organolead Perovskite Films. Sci. Rep. 7, 14760 (2017).

45. D'Innocenzo, V. et al. Excitons versus free charges in organo-lead tri-halide perovskites. Nat. Commun. 5, 3586 (2014).

46. Mooney, J. \& Kambhampati, P. Get the basics right: Jacobian conversion of wavelength and energy scales for quantitative analysis of emission spectra. Journal of Physical Chemistry Letters 4, 3316-3318 (2013).

47. Richter, J. M. et al. Ultrafast carrier thermalization in lead iodide perovskite probed with twodimensional electronic spectroscopy. Nat. Commun. 8, 1-7 (2017).

48. Hintermayr, V. A., Polavarapu, L., Urban, A. S. \& Feldmann, J. Accelerated Carrier Relaxation through Reduced Coulomb Screening in Two-Dimensional Halide Perovskite Nanoplatelets. ACS Nano 12, 45 (2018).

49. Todd, S. B. et al. Detection of Rashba spin splitting in 2D organic-inorganic perovskite via 
precessional carrier spin relaxation. APL Mater. 7, (2019).

50. Nitta, J. Semiconductor spintronics. NTT Tech. Rev. 2, 31-36 (2004).

51. Teng, L. H., Zhang, P., Lai, T. S. \& Wu, M. W. Density dependence of spin relaxation in GaAs quantum well at room temperature. EPL 84, (2008).

52. Dal Conte, S. et al. Ultrafast valley relaxation dynamics in monolayer MoS2 probed by nonequilibrium optical techniques. Phys. Rev. B - Condens. Matter Mater. Phys. 92, 235425 (2015).

53. Tan, Z.-K. et al. Bright light-emitting diodes based on organometal halide perovskite. Nat. Nanotechnol. 9, 1-6 (2014).

54. Wang, N. et al. Perovskite light-emitting diodes based on solution-processed self-organized multiple quantum wells. Nat. Photonics 10, 699-704 (2016).

55. Zhao, B. et al. High-efficiency perovskite-polymer bulk heterostructure light-emitting diodes. Nat. Photonics 12, 1-10 (2018).

56. Chen, K., Gallaher, J. K., Barker, A. J. \& Hodgkiss, J. M. Transient grating photoluminescence spectroscopy: An ultrafast method of gating broadband spectra. J. Phys. Chem. Lett. 5, 1732-1737 (2014). 